QUARTERLY OF APPLIED MATHEMATICS

VOLUME LXX, NUMBER 1

MARCH 2012, PAGES 171-180

S 0033-569X(2011)01247-1

Article electronically published on August 29, 2011

\title{
ON THE INFLUENCE OF DAMPING IN HYPERBOLIC EQUATIONS WITH PARABOLIC DEGENERACY
}

\author{
BY \\ KATARZYNA SAXTON (Department of Mathematical Sciences, Loyola University, New Orleans, \\ Louisiana 70118) \\ AND \\ RALPH SAXTON (Department of Mathematics, University of New Orleans, New Orleans, Louisiana \\ $70148)$
}

Abstract. This paper examines the effect of damping on a nonstrictly hyperbolic $2 \times 2$ system. It is shown that the growth of singularities is not restricted as in the strictly hyperbolic case where dissipation can be strong enough to preserve the smoothness of small solutions globally in time. Here, irrespective of the stabilizing properties of damping, solutions are found to break down in finite time on a line where two eigenvalues coincide in state space.

1. Introduction. In this paper, we consider a $2 \times 2$ damped, nonstrictly hyperbolic system of pde's in one space dimension:

$$
\begin{array}{r}
e_{t}-p_{x}=0, \\
p_{t}-\mathrm{G}(e)_{x}=-b p,
\end{array}
$$

where $b>0$ is a constant. This means that along a curve $\Sigma$ in $\mathbb{R}^{2}$, the associated characteristic speeds coincide. An overview of problems associated with undamped, nonstrictly hyperbolic systems is discussed in [5], and there are many results concerning the influence of damping on global existence and singularity formation in solutions to strictly hyperbolic systems. It is well known that in the latter case, for sufficiently smooth, small amplitude initial data, solutions persist globally in time, 9], while solutions generally blow up in finite time for large amplitude data, 14.

In the present work, the two eigenvalues, $\lambda_{1}(e) \leq \lambda_{2}(e)$, of the matrix $A=\operatorname{grad} F$, $F=(-p,-\mathrm{G}(e))$, are distinct if $e \neq 0$ and coincide on $\Sigma=\left\{(e, p) \subset \mathbb{R}^{2}: e=0\right\}$. The system loses strict hyperbolicity at $e=0$, where $A$ has only a single eigenvector, which means that the system exhibits parabolic degeneracy on $\Sigma$, 4].

Received September 6, 2010.

2010 Mathematics Subject Classification. Primary 35L45, 35L67.

E-mail address: saxton@loyno.edu

E-mail address: rsaxton@uno.edu

(C)2011 Brown University

Reverts to public domain 28 years from publication 
This type of problem occurs in low temperature thermal wave propagation if the heat capacity, $c_{V}$, has an integrable singularity at a temperature $\vartheta=\vartheta_{\lambda}, \vartheta$ denoting absolute temperature. We briefly describe the setting.

The balance of energy reads

$$
\varepsilon(\vartheta)_{t}+q_{x}=0,
$$

where $\varepsilon$ is the internal energy, $\varepsilon^{\prime}(\vartheta)=c_{V}(\vartheta), q$ represents heat flux, $q=-\alpha(\vartheta) p$, and for small $\left|\vartheta-\vartheta_{\lambda}\right|, c_{V}$ satisfies a condition such as $([12])$

$$
c_{V}(\vartheta)=c\left|\vartheta-\vartheta_{\lambda}\right|^{-\nu}, \quad c>0, \quad \nu \in(0,1) .
$$

The variable $p$ denotes an internal parameter which obeys an associated evolution equation of the form

$$
p_{t}-g(\vartheta)_{x}=f(\vartheta) p .
$$

Using the above hypothesis for $q$ together with equation (1.5) is equivalent to having $q$ depend on the history of the temperature gradient, [11. The constitutive functions $\alpha(\vartheta)>0, g^{\prime}(\vartheta)>0, f(\vartheta)<0$ can be derived from measurable equilibrium properties of $c_{V}(\vartheta)$, the heat conductivity $K(\vartheta)$, and the second sound velocity, $U_{E}(\vartheta)$ (see [10, 11] for details).

The system (1.3), (1.5) has characteristic speeds

$$
\lambda_{1,2}=\frac{1}{2}\left(-\frac{\alpha^{\prime} p}{c_{V}} \pm \sqrt{\left(\frac{\alpha^{\prime} p}{c_{V}}\right)^{2}+4 \frac{\alpha g^{\prime}}{c_{V}}}\right),
$$

where, for finite $p, \lambda_{1}\left(\vartheta_{\lambda}, p\right)=\lambda_{2}\left(\vartheta_{\lambda}, p\right)=0$, by (1.4).

We transform (1.3), (1.5) into (1.1), (1.2) by setting $e=\varepsilon(\vartheta)-\varepsilon\left(\vartheta_{\lambda}\right)$. Then $\vartheta=$ $\vartheta(e)=\varepsilon^{-1}\left(e+\varepsilon_{\lambda}\right)$, where $\varepsilon_{\lambda}=\varepsilon\left(\vartheta_{\lambda}\right)$, which gives 1

$$
\begin{gathered}
e_{t}-(\alpha(e) p)_{x}=0, \\
p_{t}-\mathrm{G}(e)_{x}=f(e) p .
\end{gathered}
$$

Assuming that $\alpha(e)=1, f(e)=-b$, and writing $c_{V}$ in terms of the variable $e$ as

$$
c_{V}(e)=c m^{m-1}|e|^{1-m}, \quad m=\frac{1}{1-\nu}>1,
$$

we recover (1.1) and (1.2), with the properties that $\mathrm{G}^{\prime}(0)=0$ and $\lambda_{1}=\lambda_{2}=0$ at $e=0$.

In the next sections, we consider (1.1), (1.2) under the following set of hypotheses:

H 1. i) $\mathrm{G}^{\prime}(z)>0$, ii) $z \mathrm{G}^{\prime \prime}(z)>0$ and iii) $\mathrm{G}^{\prime \prime \prime}(z)>0, \quad z \neq 0$;

H 2. i) $G^{\prime}(0)=G^{\prime \prime}(0)=0$ and ii) $G^{\prime \prime \prime}(0)>0$.

2. Damping in the strictly and nonstrictly hyperbolic cases. In hyperbolic systems without damping, globally smooth solutions generally fail to exist since singularities can form in finite time. However, the fact that the presence of damping in strictly hyperbolic systems may prevent certain singularities from forming has been known since the pioneering work of Nishida, 9]. In this paper, we investigate whether this effect persists in regions where strict hyperbolicity is lost. In order to contrast these two cases we begin by examining weakly discontinuous waves, or acceleration waves.

\footnotetext{
${ }^{1} \mathrm{An}$ asymptotic analysis of (1.7), (1.8) can be found in 8 in the case of nonsingular specific heat.
} 
Suppose the curve, $\mathrm{S}=\left\{(x, t) \in \mathbb{R}^{2}: x=\varphi(t)\right\}$, represents a wave front for (1.1), (1.2), ahead of which we have an equilibrium state, $(e, p)=\left(e^{+}, 0\right), e^{+}=$constant, and behind which there lies an unknown, perturbed state $(e(x, t), p(x, t))$. The speed of propagation of the front is defined by $v(t)=\dot{\varphi}(t)$. A function $\phi(x, t)$ may exhibit a finite jump

$$
[\phi](\varphi(t), t)=\phi\left(\varphi^{-}(t), t\right)-\phi\left(\varphi^{+}(t), t\right)
$$

across the front, and its derivative is given by

$$
\frac{d}{d t}[\phi]=\left[\phi_{t}\right]+v\left[\phi_{x}\right] .
$$

$\mathrm{S}$ is called a weakly discontinuous wave if $e(x, t)$ and $p(x, t)$ are continuous across $\mathrm{S}$, but their derivatives need not be. It follows from (2.2) that

$$
\left[e_{t}\right]=-v\left[e_{x}\right], \quad\left[p_{t}\right]=-v\left[p_{x}\right] .
$$

Evaluating (1.1), (1.2) across S and using (2.3) gives an explicit formula for the speed of propagation,

$$
v(t)=\lambda_{2}\left(e^{+}\right)=\sqrt{\mathrm{G}^{\prime}\left(e^{+}\right)}=\text {const },
$$

where $\lambda_{1}(e)=-\sqrt{\mathrm{G}^{\prime}(e)}$ and $\lambda_{2}(e)=\sqrt{\mathrm{G}^{\prime}(e)}$ are the eigenvalues for the system (1.1), (1.2).

Next, we denote by $r$ the amplitude of a jump discontinuity in $e_{x}$,

$$
r(t) \equiv\left[e_{x}\right](\varphi(t), t) .
$$

This amplitude satisfies the equation ([2])

$$
2 v^{2} \frac{d r}{d t}+\mathrm{G}^{\prime \prime}\left(e^{+}\right) v r^{2}+b v^{2} r=0,
$$

which can be derived by differentiating both (1.1) and (1.2) with respect to time and using the identities

$$
\frac{d r}{d t}=\left[e_{x t}\right]+v\left[e_{x x}\right], \quad \frac{d\left[p_{t}\right]}{d t}=v^{2} \frac{d r}{d t} .
$$

CASE I. $e^{+} \neq 0$. Then $v \neq 0$ by hypothesis $\mathrm{H} 1$, i) and equation (2.4). Thus (2.6) is equivalent to the equation

$$
\frac{d r}{d t}+A r^{2}+B r=0,
$$

where $A=\frac{\mathrm{G}^{\prime \prime}\left(e^{+}\right)}{2 v}=\frac{\mathrm{G}^{\prime \prime}\left(e^{+}\right)}{2 \sqrt{\mathrm{G}^{\prime}\left(e^{+}\right)}}, B=\frac{b}{2}>0$.

A simple analysis of this Bernoulli equation shows that there exists a threshold below which the amplitude of solutions stays bounded for all time. The main conclusions can be summarized by the following.

1. If $A r_{0}>0$, where $r_{0}=r(0)=\left[e_{x}(x, 0)\right]$, then $\lim _{t \rightarrow \infty} r(t)=0$.

2. If $A r_{0}<0$, then $r(t)$ blows up in finite time only if $B+A r_{0}<0$; for $B+A r_{0}>0, \lim _{t \rightarrow \infty} r(t)=0$.

3. If $A=0$, equation (2.7) is linear, and again $\lim _{t \rightarrow \infty} r(t)=0$.

We note that $A=\left(\nabla \lambda_{2} \cdot \mathbf{d}\right)\left(e^{+}\right)$, where $\mathbf{d}$ is a right eigenvector corresponding to the eigenvalue $\lambda_{2}$. If $A=0$, the $\lambda_{2}$ characteristic is linearly degenerate at $e=e^{+}$. 
CASE II. $e^{+}=0$. Then $v=0$ by hypothesis $\mathrm{H}$ 2, i) and equation (2.4).

Now (2.6) no longer provides the evolution of the amplitude $r$ along $\mathrm{S}=\left\{(x, t) \in \mathbb{R}^{2}\right.$ : $x=\varphi(t), \varphi(t)=$ constant $\}$. The system (1.1), (1.2) is not strictly hyperbolic at $e=0$ since $\lambda_{1}(0)=\lambda_{2}(0)=0=v(t)$ and $\mathrm{S} \equiv \Sigma$.

As in Case I, we first differentiate (1.1), (1.2) with respect to $t$, but then also with respect to $x$. Evaluating across $\mathrm{S}$, we have

$$
\left[e_{x t t}\right]=\mathrm{G}^{\prime \prime \prime}(0)\left[e_{x}\right]^{3}+3 \mathrm{G}^{\prime \prime}(0)\left[e_{x}\right]\left[e_{x x}\right]+\mathrm{G}^{\prime}(0)\left[e_{x x}\right]-b\left[e_{x t}\right]=0,
$$

where the following relations have been used:

$$
\begin{gathered}
\frac{d}{d t}\left[e_{x}\right]=\left[e_{x t}\right], \\
\frac{d}{d t}\left[e_{x t}\right]=\frac{d^{2}}{d t^{2}}\left[e_{x}\right]=\left[e_{x t t}\right] .
\end{gathered}
$$

Recalling $\mathrm{H} 2 \mathrm{i}$ ) and ii), equation (2.8) yields

$$
\ddot{r}+b \dot{r}-a r^{3}=0 \text {, where } a=\mathrm{G}^{\prime \prime \prime}(0) .
$$

If the coefficient, $a$, of the cubic term is zero, then the equation is $\operatorname{linear}_{t \rightarrow \infty} \lim _{t \rightarrow \infty} r(t)=$ const, and blowup does not occur. This coefficient is zero if $\left(\nabla \lambda_{2} \cdot d\right)(0)=\frac{\mathrm{G}^{\prime \prime}(0)}{2 \sqrt{\mathrm{G}^{\prime}(0)}}=0$, i.e. if the system once more exhibits linear degeneracy. To prove this, we employ the following basic result.

Proposition 2.1. Let $f \in C^{1}, g \in C^{0}$ be defined in a neighborhood of the origin with range $\{f\} \subseteq$ domain $\{g\} \cap \operatorname{domain}\left\{g^{\prime}\right\}$ and satisfy $\left.f(0)=f^{\prime} 0\right)=g(0)=0$, and suppose that the limit

$$
\mathcal{M} \equiv \lim _{x \rightarrow 0} \frac{f^{\prime \prime}(x)}{(g \circ f)(x) \cdot\left(g^{\prime} \circ f\right)(x)}
$$

exists. Then $\mathcal{L} \equiv \lim _{x \rightarrow 0} \frac{f^{\prime}(x)}{(g \circ f)(x)}$ also exists, and satisfies $\mathcal{L}^{2}=\mathcal{M}$.

Proof. Setting $\mathcal{F}(x)=f^{\prime}(x)$ and $\mathcal{G}(x)=(g \circ f)(x)$ in the general form of L'Hôpital's rule leads to

$$
\lim _{x \rightarrow 0} \frac{f^{\prime}(x)}{(g \circ f)(x)}=\lim _{x \rightarrow 0} \frac{f^{\prime \prime}(x)}{(g \circ f)(x)\left(g^{\prime} \circ f\right)(x) \cdot \frac{f^{\prime}(x)}{(g \circ f)(x)}},
$$

from which the result follows.

In particular, the case where $g(y)=\sqrt{y}$ shows that for $f \geq 0$,

$$
\lim _{x \rightarrow 0} f^{\prime \prime}(x)=\lim _{x \rightarrow 0} \frac{f^{\prime}(x)^{2}}{2 f(x)}
$$

provided the limit exists. The statement before the proposition follows on taking $f=G^{\prime}$ in (2.10).

Whether assuming strict or nonstrict hyperbolicity, both equations for $r(t)$ were derived under the condition $\left[e_{x}\right](., 0) \neq 0$. We next show, briefly, that equation (2.9) is also relevant to the case of smooth initial data.

Consider again (1.1), (1.2) under hypotheses $\mathrm{H} 1$ and $\mathrm{H} 2$, for smooth data

$$
e(x, 0)=e_{0}(x), \quad p(x, 0)=p_{0}(x),
$$


with

$$
e_{0}(\beta)=p_{0}^{\prime}(\beta)=0 \quad \text { and } \quad(x-\beta) e_{0}(x)>0 \quad \text { for } \quad x \neq \beta .
$$

Lemma 2.2. Let $T>0$ and let $(e, p)$ be a bounded solution to (1.1), (1.2) in $C^{2}\left([0, T] ; C^{0}(\mathbb{R})\right) \cap C^{0}\left([0, T] ; C^{2}(\mathbb{R})\right) \times C^{1}\left([0, T] ; C^{1}(\mathbb{R})\right), 0 \leq t \leq T$. Further, suppose that $\mathrm{G}^{\prime \prime}$ is locally Lipschitz continuous and that $\left.\mathrm{H} 2 \mathrm{i}\right)$ holds. Then $e(\beta, t)=0$ for $0 \leq t \leq T$.

Proof. First, by differentiating (1.1) with respect to $t$ and (1.2) with respect to $x$, we obtain the second-order equation

$$
e_{t t}=\mathrm{G}^{\prime \prime}(e) e_{x}^{2}+\mathrm{G}^{\prime}(e) e_{x x}-b e_{t} .
$$

Integrating this in time at $x=\beta$ leads to

$$
e_{t}(\beta, t)=\int_{0}^{t}\left(\mathrm{G}^{\prime \prime}(e) e_{x}^{2}+\mathrm{G}^{\prime}(e) e_{x x}\right)(\beta, \tau) d \tau-b e(\beta, t)
$$

by (2.12), and we have for $b>0$,

$$
e(\beta, t)=\frac{1}{b} \int_{0}^{t}\left(1-e^{b(\tau-t)}\right)\left(\mathrm{G}^{\prime \prime}(e) e_{x}^{2}+\mathrm{G}^{\prime}(e) e_{x x}\right)(\beta, \tau) d \tau .
$$

Due to $\mathrm{H} 2 \mathrm{i}$ ) and the Lipschitz continuity of $\mathrm{G}^{\prime \prime}$, we conclude that for $t<T$,

$$
|e(\beta, t)| \leq c \int_{0}^{t}|e(\beta, \tau)| d \tau,
$$

where $c$ depends on the sizes of $e_{x}$ and $e_{x x}$. Grönwall's inequality then implies that $e(\beta, t)=0$ for $t<T$.

The result means that the line $x=\beta$ is characteristic, with $\lambda_{1}(0)=\lambda_{2}(0)=0$, at least until the solution breaks down. Next, differentiating (2.13) in $x$ at $x=\beta$ gives

$$
e_{x t t}(\beta, t)=\mathrm{G}^{\prime \prime \prime}(0) e_{x}(\beta, t)^{3}-b e_{x t}(\beta, t) .
$$

Choosing $r(t)=e_{x}(\beta, t)$ lets us write equation (2.17), exactly as (2.9).

3. Phase plane analysis. In this section we examine solutions to (1.1), (1.2) on $\Sigma=\left\{(e, p) \subset \mathbb{R}^{2}: e=0\right\}$ by first performing a brief analysis of local stability and then obtaining an important a priori property for (2.9).

Since the change of variables $t=b^{-1} \tau, r=b a^{-1 / 2} R$, lets us write an equation of the same type as (2.9) for $R(\tau)$, but with $a=b=1$, we will revert to the original notation and consider the basic second-order equation

$$
\ddot{r}+\dot{r}-r^{3}=0,
$$

with data

$$
r(0)=r_{0}, \quad \dot{r}(0)=r_{1},
$$

or the equivalent first-order system,

$$
\begin{array}{r}
\dot{r}=s, \\
\dot{s}=-s+r^{3} .
\end{array}
$$


Since the linearized version of this system has only zero and negative eigenvalues, we use the center manifold theorem, [1, to investigate the stability of the origin. Introducing new variables, $r=x+y$ and $s=-y$, the system (3.3), (3.4) takes the canonical form

$$
\begin{array}{r}
\dot{x}=(x+y)^{3}, \\
\dot{y}=-y-(x+y)^{3} .
\end{array}
$$

The function $f(x, y)=(x+y)^{3}$ satisfies $f(0,0)=f_{x}(0,0)=f_{y}(0,0)=0$, and a onedimensional center manifold for (3.5), (3.6) can be represented as a graph $W^{c}=\{(x, y)$ : $\left.y=h(x) ; h(0)=h^{\prime}(0)=0\right\}$ with $h(x)$ defined over a local neighborhood of $x=0$, tangent to the linear subspace $E^{c}=\{(x, y): y=0\}$ in $\mathbb{R}^{2}$. Substituting $y=h(x)$ in (3.5) gives

$$
\dot{x}=(x+h(x))^{3} .
$$

Local stability, asymptotic stability or instability of (3.5), (3.6) at $(x, y)=(0,0)$ follows from that of (3.7) at $x=0([1,3])$.

Substituting $y=h(x)$ in (3.6) and using (3.7) leads to the equation for the center manifold,

$$
\left(1+h^{\prime}(x)\right)(x+h(x))^{3}+h(x)=0 .
$$

A polynomial approximation $\phi(x)$ can be obtained for $h(x)$.

Proposition 3.1. Let $\phi(x)=-x^{3}+6 x^{5}$. Then $|h(x)-\phi(x)|=O\left(|x|^{7}\right)$, as $|x| \rightarrow 0$.

The proof of this proposition comes from well-known arguments. As a result, equation (3.7) can be written as

$$
\dot{x}=x^{3}-3 x^{5}+O\left(|x|^{7}\right) .
$$

Instability of the zero solution of (3.9) is now immediate, from which instability of (3.7) and that of the system (3.5), (3.6) (respectively (3.3), (3.4)) follows.

For completeness, we also describe the local stable manifold. Since this will be given in $(r, s)$ variables, we note that the center manifold approximation can be written as $s \approx r^{3}$ in these coordinates.

The local stable manifold can be represented as the graph $W_{l o c}^{s}=\{(r, s): s=$ $\left.\tilde{h}(r) ; \tilde{h}(0)=0, \tilde{h}^{\prime}(0)=-1\right\}$ with $\tilde{h}(r)$ defined over a local neighborhood of $r=0$, tangent to the linear subspace $E^{s}=\{(r, s): s=-r\}$ in $\mathbb{R}^{2}$. Since $s \frac{d s}{d r}=r^{3}-s$ by (3.3), (3.4), we obtain the local approximation

$$
\tilde{h}(r)=-r-\frac{1}{3} r^{3}+O\left(|r|^{5}\right)
$$

We will denote by $s=\tilde{s}(r)(\tilde{s}(0)=0)$ the corresponding global stable manifold $W^{s}$ (see [3]) whose properties are used below.

For the remaining discussion, owing to the invariance of (3.3), (3.4) under the transformation $r \rightarrow-r, s \rightarrow-s$, we will restrict attention to the half-plane $(r, s) \in \mathbb{R} \times[0, \infty)$ which we divide into

$$
\begin{aligned}
& U^{+}=\{(r, s) \in \mathbb{R} \times[0, \infty): \hat{\mathcal{E}}(r, s)>0\}, \\
& U^{-}=\{(r, s) \in \mathbb{R} \times[0, \infty): \hat{\mathcal{E}}(r, s)<0\},
\end{aligned}
$$


with boundary

$$
U^{0}=\{(r, s) \in \mathbb{R} \times[0, \infty): \hat{\mathcal{E}}(r, s)=0\}
$$

where

$$
\hat{\mathcal{E}}(r, s)=\frac{1}{2} s^{2}-\frac{1}{4} r^{4} .
$$

Defining

$$
\mathcal{E}(t)=\hat{\mathcal{E}}(r(t), \dot{r}(t)) \text { for } t>0, \quad \mathcal{E}(0)=\frac{1}{2} r_{1}^{2}-\frac{1}{4} r_{0}^{4}
$$

gives

$$
\dot{\mathcal{E}}=-\dot{r}^{2}
$$

by (3.1), and so

$$
\mathcal{E}(t)=\mathcal{E}(0)-\int_{0}^{t} \dot{r}^{2}(\tau) d \tau
$$

or equivalently

$$
\hat{\mathcal{E}}(r, \hat{s}(r))=\hat{\mathcal{E}}\left(r_{0}, r_{1}\right)-\int_{r_{0}}^{r} \hat{s}(\rho) d \rho,
$$

where $\hat{s}(r(t)) \equiv s(t)$.

Lemma 3.2. Let $r \rightarrow \tilde{s}(r)(\tilde{s}(0)=0)$ represent the stable manifold, $W^{s}$ (for positive $\tilde{s}$ ) and denote by $\hat{s}(r)(\hat{s}(0) \neq 0)$ any other solution curve. Then

i) $(r, \tilde{s}(r)) \in U^{+}$with $r(t) \leq 0$ for all $t \geq 0$;

ii) if $\left(r_{0}, \hat{s}\left(r_{0}\right)\right) \in U^{+}$, then there exists $r_{*}=r\left(t_{*}\right), t_{*}<\infty$, such that $\left(r_{*}, \hat{s}\left(r_{*}\right)\right) \in U^{0}$, and $(r(t), s(t)) \in U^{-}$for all $t>t_{*}$.

Proof. i) Let $\left(r_{0}, r_{1}\right), r_{1}>0$, lie on the global stable manifold, $W^{s}$. Then at $r=0$, (3.18) gives

$$
\tilde{\mathcal{E}}\left(r_{0}, r_{1}\right)=\int_{r_{0}}^{0} \tilde{s}(\rho) d \rho=\int_{0}^{\infty} \dot{r}^{2}(\tau) d \tau>0
$$

so $\left(r_{0}, r_{1}\right) \in U^{+}$. Further, $\tilde{s}(\rho)>0$ except at $\rho=0$ since, if $\tilde{s}(\rho) \rightarrow 0$ with $\rho \neq 0$, then $\tilde{\mathcal{E}}(\rho, 0)<0$ by (3.14), contradicting the fact that for any $\tilde{t}>0$ at which $\tilde{s}(\rho(\tilde{t}))=0$, $\tilde{\mathcal{E}}(\rho(\tilde{t}), 0)=\int_{\tilde{t}}^{\infty} \dot{r}^{2}(\tau) d \tau>0$. Therefore $r_{0}<0$ by (3.19), which gives i) since $\left(r_{0}, r_{1}\right)$ was an arbitrary point on $W^{s}$.

ii) Let $\Delta(r)$ denote the difference between the values of $s$ on the curves $s=\hat{s}(r)$, with $r_{1}=\hat{s}\left(r_{0}\right),\left(r_{0}, r_{1}\right) \in U^{+} \backslash W^{s}$, and $s=\stackrel{s}{s}(r)=\frac{1}{\sqrt{2}} r^{2}$, representing $U_{0}$. Calculating that $\frac{d \Delta}{d r}=\frac{r^{3}}{\hat{s}}-(1+\sqrt{2} r)<-1$ gives the inequality $\Delta(r)<\Delta\left(r_{0}\right)-\left(r-r_{0}\right)$ in $U^{+}$. If $\Delta(r)>0$ in $U^{+}$for all $r>r_{0}$, then $\Delta\left(r_{0}\right)>r-r_{0}$, which is impossible. Therefore $\Delta(r) \rightarrow 0$ at some finite value, $r=r_{*}$. Thus, since $\dot{\mathcal{E}}(t)<0$ for all $t>0$, every solution curve from $U^{+} \backslash W^{s}$ intersects $U^{0} \backslash\{(0,0)\}$ then crosses over $U^{0}$ to $U^{-}$.

Finally we verify that if $r\left(t_{*}\right)=r_{*}$, then $t_{*}<\infty$. By uniqueness of $W^{s}$, it is easy to see that $\hat{s}(r)$ has a (strictly positive) least value at some point $\left(r_{m}, s_{m}\right) \in U^{+} \cup U_{0}$, where $s_{m}=\hat{s}\left(r_{m}\right)$. If the curve $(r, \hat{s}(r))$ starts below $W^{s}$, then $\left(r_{m}, s_{m}\right) \in U_{0}$, while if $(r, \hat{s}(r))$ begins above $W^{s}$, then either $\left(r_{m}, s_{m}\right) \in\left\{(r, s) \in U^{+}: s=r^{3}\right\}$ or $\left(r_{m}, s_{m}\right)=\left(r_{0}, r_{1}\right)$. So we have

$$
\hat{s}(r) \geq s_{m}>0, \text { for all }(r, \hat{s}(r)) \in U^{+} \cup U_{0} .
$$


Using (3.18) at $r=r_{*}$ where $\hat{\mathcal{E}}\left(r_{*}, \hat{s}\left(r_{*}\right)\right)=0$, together with (3.20), then leads to the inequality

$$
\hat{\mathcal{E}}\left(r_{0}, r_{1}\right)=\int_{r_{0}}^{r_{*}} \hat{s}(\rho) d \rho=\int_{0}^{t_{*}} \dot{r}^{2}(\tau) d \tau>\left(s_{m}\right)^{2} t_{*},
$$

which implies that $t_{*}<\infty$.

4. Singularity formation. We have shown that, with the exception of solutions lying on the one-dimensional global stable manifold $W^{s}$, all solutions starting with initial data having $\mathcal{E}(0) \geq 0$ eventually satisfy $\mathcal{E}(t)<0$ for all $t>t_{*}$, where $t_{*}<\infty$. The only such solutions which satisfy $\mathcal{E}(t) \geq 0$ for all $t \geq 0$ are those lying on the stable manifold.

In this section, we use a 'concavity method' approach ([6], [7]) to demonstrate finite time blowup of solutions to (3.1). Since solutions to the (equivalent) autonomous system (3.3), (3.4) define a local one-parameter group, $(r, s)\left(t_{2}+t_{1} ;\left(r_{0}, r_{1}\right)=(r, s)\left(t_{2} ;\left((r, s)\left(t_{1}\right.\right.\right.\right.$; $\left.\left.\left(r_{0}, r_{1}\right)\right)\right)$, where $(r, s)\left(t ;\left(r_{0}, r_{1}\right)\right)$ denotes a solution such that $(r, s)\left(0 ;\left(r_{0}, r_{1}\right)\right)=\left(r_{0}, r_{1}\right)$. This means that we may, for convenience of notation, continue to represent trajectories in $U^{-}$(see (3.12) ) as starting from $t=0$.

Theorem 4.1. Let $r(t)$ be a solution to the initial value problem (3.1), (3.2), defined over a maximal interval of existence, $t \in\left[0, t^{*}\right)$, and suppose that $\mathcal{E}(0)<0$. Then $t^{*}<\infty$ and $\lim _{t \rightarrow t^{*}-}|r(t)|=\infty$.

Proof. Writing equation (3.17) as

$$
\frac{1}{4} r^{4}(t)=\frac{1}{2} \dot{r}^{2}(t)-\mathcal{E}(0)+\int_{0}^{t} \dot{r}^{2}(\tau) d \tau
$$

we introduce the expression

$$
H(t)=r^{2}(t)+\int_{0}^{t} r^{2}(\tau) d \tau+\left(T_{0}-t\right) r_{0}^{2}+\xi\left(T_{1}+t\right)^{2},
$$

defined for $0 \leq t<T_{0}$ ([7, 15]). We will show below that for suitable $T_{0}, T_{1}, \xi>0, H(t)$ satisfies the following properties:

$$
H(0)>0, \quad \dot{H}(0)>0, \quad \ddot{H}(t)>0,
$$

and

$$
H(t) \ddot{H}(t)-\frac{3}{2} \dot{H}^{2}(t) \geq 0 .
$$

As a consequence of (4.3) and (4.4), it follows that

$$
\frac{. .}{H^{-1 / 2}}(t) \leq 0 .
$$

Therefore, on integrating,

$$
0<H^{-1 / 2}(t) \leq \bar{\cdot} \overline{H^{-1 / 2}}(0) t+H^{-1 / 2}(0),
$$

from which

$$
H^{1 / 2}(t) \geq \frac{1}{\overline{H^{-1 / 2}}(0) t+H^{-1 / 2}(0)},
$$


and so we find that

$$
H(t) \rightarrow \infty \text { as } t \rightarrow T-, \text { where } T \leq t^{*} \equiv 2 \frac{H(0)}{\dot{H}(0)} .
$$

To conclude, we need to establish assertions (4.3) and (4.4).

On differentiating (4.2),

$$
\dot{H}(t)=2 r(t) \dot{r}(t)+2 \int_{0}^{t} r(\tau) \dot{r}(\tau) d \tau+2 \xi\left(T_{1}+t\right),
$$

and taking the second derivative and using (3.1), (4.1) gives $(4.3)_{3}$, since

$$
\ddot{H}(t)=6 \dot{r}^{2}(t)+8 \int_{0}^{t} \dot{r}^{2}(\tau) d \tau-8 \mathcal{E}(0)+2 \xi
$$

and $\mathcal{E}(0)<0$ in $U^{-}$. We next choose $\xi \in(0,-2 \mathcal{E}(0))$ so that

$$
\ddot{H}(t) \geq 6 \dot{r}^{2}(t)+8 \int_{0}^{t} \dot{r}^{2} d \tau+6 \xi .
$$

Next, choosing $T_{1}$ large enough so that (see (4.9))

$$
\dot{H}(0)=2 r_{0} r_{1}+2 \xi T_{1}>2 r_{0}^{2}
$$

gives $(4.3)_{2}$, and $(4.3)_{1}$ comes directly from (4.2). Note that $\dot{H}(t)>0$ since $\dot{H}(0)>0$ and $\ddot{H}(t)>0$. To justify (4.4), let

$$
\begin{gathered}
K(t)=r^{2}(t)+\int_{0}^{t} r^{2}(\tau) d \tau+\xi\left(T_{1}+t\right)^{2}, \\
L(t)=\frac{1}{2} \dot{H}(t), \\
M(t)=\dot{r}^{2}(t)+\int_{0}^{t} \dot{r}^{2}(\tau) d \tau+\xi
\end{gathered}
$$

(see [15]). Then, provided $r(t)$ exists over the interval $t \in\left[0, T_{0}\right)$, we have the inequality

$$
H(t)=K(t)+\left(T_{0}-t\right) r_{0}^{2} \geq K(t)
$$

and, by (4.11),

$$
\ddot{H}(t) \geq 6 M(t) .
$$

Using (4.14), (4.16) and (4.17), we calculate the expression on the left-hand side in (4.4) to obtain

$$
H(t) \ddot{H}(t)-\frac{3}{2} \dot{H}^{2}(t) \geq 6\left(K M-L^{2}\right) .
$$

A direct examination of the terms shows that $K z^{2}-2 L z+M \geq 0$ for arbitrary $z \in \mathbb{R}$, and so we arrive at (4.4) via (4.18). Finally, recalling the argument leading to the expression for the maximal time of existence, $t^{*}$, in (4.8), we observe using (4.12) and (4.2) with $t=0$, that taking $T_{0}>\frac{r_{0}^{2}+\xi T_{1}^{2}}{r_{0} r_{1}+\xi T_{1}-r_{0}^{2}}>0$ implies $t^{*}<T_{0}$

Our results show that for the case of nonstrictly hyperbolic equations, damping generally does not prevent finite time singularity formation. This differs from the strictly hyperbolic case where damping guarantees global existence of small solutions ([9]); only large amplitude data can lead to finite time breakdown ([14]). If $e_{0}(x)$ and $p_{0}(x)$ satisfy 
(2.11), (2.12) and together with $e_{0}^{\prime}(x), p_{0}^{\prime}(x)$ are sufficiently small, then singularities can form only on $\Sigma$. These singularities arise unless $\left(e_{0}^{\prime}(\beta), p_{0}^{\prime \prime}(\beta)\right)=\left(r_{0}, r_{1}\right) \in W^{s}$.

We note that for the nonstrictly hyperbolic system (1.1), (1.2), in either the damped, $b>0$, or undamped, $b=0$ (cf. [13]) cases, the following holds:

a) there is a unique codimension one stable manifold $W^{s}: s=\tilde{s}(r)$ (for $\left.b>0\right)$ and $s=\tilde{\tilde{s}}(r)$ (for $b=0$; here $\tilde{\tilde{s}}(r)=\stackrel{\circ}{s}(r)$ or $\tilde{\tilde{s}}(r)=-\stackrel{\circ}{s}(r)$ if $r \leq 0, r>0$, respectively) on which solutions decay to equilibrium;

b) in both cases, away from the stable manifold, genuine nonlinearity is responsible for finite time breakdown only if $\mathrm{G}^{\prime \prime \prime}(0)=a>0$.

\section{REFERENCES}

[1] J. Carr, Applications of centre manifold theory, Springer-Verlag New York, Inc., 1981. MR635782 $(83 \mathrm{~g}: 34039)$

[2] C. M. Dafermos, Contemporary issues in the dynamic behaviour of continuous media, LCDS Lecture Notes, Brown University, vol. 85-1, 1985.

[3] J. Guckenheimer and P. Holmes, Nonlinear oscillations, dynamical systems, and bifurcations of vector fields, Springer-Verlag New York, Inc., 1983. MR709768 (85f:58002)

[4] B. L. Keyfitz and H. C. Kranzer, Non-strictly hyperbolic systems of conservation laws: formation of singularities, Contemporary Math., 17 (1983), pp. 77-90. MR.706089 (84h:35102)

[5] B. L. Keyfitz and C. A. Mora, Prototypes for nonstrict hyperbolicity in conservation laws, Contemp. Math., Amer. Math. Soc., 255 (2000), pp. 125-137. MR.1752505 (2001d:35132)

[6] R. J. Knops, H. A. Levine and L. E. Payne, Nonexistence, instability and growth theorems for solutions to an abstract nonlinear equation with applications to elastodynamics, Arch. Rational Mech. Anal., 55 (1974), pp. 52-72. MR0364839 (51:1093)

[7] H. A. Levine, Some additional remarks on the nonexistence of global solutions to nonlinear wave equations, SIAM J. Math. Anal., 5 (1974), pp. 138-146. MR0399682 (53:3525)

[8] H. Li and K. Saxton, Asymptotic behavior of solutions to quasilinear hyperbolic equations with nonlinear damping, Quart. Appl. Math., 61 (2003), pp. 295-313. MR1976371 (2004f:35112)

[9] T. Nishida, Nonlinear hyperbolic equations and related topics in fluid dynamics, Publications Mathématiques d'Orsay, 78.02 (1978), Dépt. de Mathématique, Paris-Sud. MR0481578 (58:1690)

[10] T. Ruggeri, A. Muracchini, and L. Seccia, Shock waves and second sound in a rigid heat conductor: A critical temperature for NaF and Bi, Phys. Rev. Lett., 64 (1990), pp. 2640-2643.

[11] K. Saxton and R. Saxton, Nonlinearity and memory effects in low temperature heat propagation, Arch. Mech., 52 (2000), pp. 127-142.

[12] K. Saxton and R. Saxton, Phase transitions and aspects of heat propagation in low temperature solids, Proceedings from the International Conference on Differential Equations Hasselt, (EQUADIFF 2003), eds. F. Dumortier et al., World Scientific, 2005, pp. 1128-1130. MR2185197

[13] R. Saxton, Blow-up at the Boundary of Solutions to Nonlinear Evolution Equations, "Evolution Equations", eds. G. Ferreyra, G. R. Goldstein and F. Neubrander, Lecture Notes in Pure and Applied Mathematics, 168 (1994), pp. 383-392. MR1300444 (95i:35025)

[14] M. Slemrod, Global existence, uniqueness, and asymptotic stability of classical smooth solutions in one-dimensional nonlinear thermoelasticity, Arch. Rat. Mech. Anal., 76 (1981), pp. 97-133. MR629700 (83c:73056)

[15] Y. Wang, Finite time blow-up results for the damped wave equations with arbitrary energy in an inhomogeneous medium, Arxiv preprint math/0702190v1 (2007). 